\title{
A Retrospective Case Study Report of the Projectification and Quick Wins during the COVID-19 Pandemic in the Governmental Healthcare Sector: Lessons Learned from Al-Ahsa, Saudi Arabia
}

\author{
Adnan Ahmed Alsaqer ${ }^{\mathrm{a}}$ Asaad Abdulrahman Abduljawad ${ }^{\mathrm{b}}$ \\ Hatim Sid Ahmed Mohammed Mustafa ${ }^{a}$ Maha Salam Mohammad ${ }^{a}$ \\ ${ }^{a}$ Alhasa Health cluster, Al-Ahsa, Saudi Arabia; ${ }^{b}$ College of Health Sciences, University of Umm Alqura Health \\ Sciences Center, Mekkah, Saudi Arabia
}

\section{Keywords}

Projectification - Project constrains - COVID-19 - Alignment · Enterprise integration

\begin{abstract}
Background: The COVID-19 pandemic is one of the most challenging disasters healthcare institutions are facing, requiring healthcare systems to balance meeting general healthcare needs with those caused by the pandemic. Healthcare systems were not designed to deal with this crisis: an unpredictable, large-scale challenge that has required urgent resource mobilization, affecting whole populations. However, there has also been no time to debate systems' abilities to deal with the crisis, not least because the pandemic opposes the general direction of these systems. The urgent need for new fast-track, quick-win projects was necessary because we were time-constrained. We also needed an effective approach - which globally known project management tools could be efficiently applied? The widely accepted Project Management Institute (PMI) model and the popular Six Sigma Define, Measure, Analyze, Implement,
\end{abstract}

Control (DMAIC) approach were two reasonable methodologies to select, so we aimed to modify these tools to achieve quick results. An initial needs assessment of the pandemic's challenges required careful consideration of our high-impact healthcare facilities' ability to meet those challenges. Our assessment concluded that ability was limited, and as Lean Six Sigma DMAIC principles are more effectively applicable to quality improvement initiatives, the combined application of DMAIC and PMI principles, which enable robust and stable project initiation, governance, enterprise engagement, and measurable quality deliverables, enabled us to focus on structured project management and quality outcomes. Methods: This modified approach was applied to the 10 main Ministry of Health $(\mathrm{MOH})$ hospitals and 2 specialized care centers in the Al-Ahsa region, to design and initiate 40 mini-projects that could yield quick wins. This study was designed to measure the value of applying these tools and learn lessons retrospectively. Results: The successful ability to eliminate identified healthcare challenges within 5 weeks of launching the mini-projects, meeting an average of $74 \%$ of planned baseline targets.

(c) 2022 The Author(s)

Published by S. Karger AG, Basel karger@karger.com www.karger.com/sjh

Karger $\stackrel{\text { ' }}{5}$

BOPEN ACCESS
(C) 2022 The Author(s)

Published by S. Karger AG, Basel

This is an Open Access article licensed under the Creative Commons Attribution-NonCommercial-4.0 International License (CC BY-NC) (http://www.karger.com/Services/OpenAccessLicense), applicable to the online version of the article only. Usage and distribution for commercial purposes requires written permission.
Correspondence to:

Adnan Ahmed Alsaqer, aalsaqer@moh.gov.sa 


\section{Introduction}

A project is defined as a temporary endeavor undertaken to create a unique product, service, or result [1]. However, projectification is designed to address challenges to a real-world project, resulting in the adding of value, the improvement of products, and improving services - in this case, the continuity with the COVID-19 crisis and the resultant variabilities. Projectification is a process; it is a path taken toward increased orientation to, and use of, projects and toward formalization of project management and the project form of organizing, rather than a one-time event [2].

According to Hodgson et al. [2], the public sector in the recent years has increased projectification in Europe and around the world, and there is a shift in the delivery of services to increase flexibility and innovation. Project management has been proven to be the ideal approach to meet the challenges of operational improvement to bring about better performance in healthcare systems performance. Among all Organization for Economic Cooperation and Development Countries (OECD) members, only Quebec in Canada has really made use of "the project" for the tactical leverage of its healthcare system, focusing on the areas of clinical care, public health services, and social services [3].

Currently, as with many other sectors, the healthcare industry is struggling with the challenges created by the COVID-19 pandemic. The healthcare sector is the primary defense line that protects the whole community and provides care for all. The impact of the pandemic, alongside the responsibility of healthcare, was an eye opener and called for the attention of the leaders of healthcare organizations.

Furthermore, there was the significant challenge of time constraints in responding and collaborating effectively by healthcare leaders who needed to search for the proper management approach for getting secured workflows in different circumstances such as, updating policies, clinical protocols, management of personal protective equipment (PPE), continuity of care for chronic diseases, and patients' transportation [4].

In fact, there is a global scarcity of published articles related to healthcare project management. In the period from 1979 to 2017, there were just 81 articles published in peer-reviewed journals, and $93 \%$ of these published papers were not in project management journals such as the International Journal of Project Management, Project Management Journal, and International Journal of Managing Project in Business system [3].

Projectification and Quick Wins during the COVID-19 Pandemic
The researchers' aim was to report the activities that took place, how teams were able to cope, and to describe the characteristics of projectification and the findings from the Project Management Institute (PMI)-Define, Measure, Analyze, Implement, Control (DMAIC) approach in the healthcare sector to control the COVID-19 pandemic, which happened and took place in the Kingdom of Saudi Arabia's Al-Ahsa region in this retrospective study. Since the first case appeared in the Kingdom, skill sets such as quality improvement have been indispensable in battling the pandemic in Ministry of Health $(\mathrm{MOH})$ hospitals in general and in Al-Ahsa hospitals specifically. The invaluable skills of healthcare workers and providers have been applied in task forces to manage the outbreak. These teams worked proactively to mitigate the harm caused by this unpredictable and unique pathogen.

The $\mathrm{MOH}$ has always been committed to providing contingency planning with clear authority indications, responsibilities, and pathways in case of emergencies, disasters, and endemics by prioritizing and producing guidance about triage, the deployment of accessible testing, physical and human resource management, surge capacity, and sharing algorithms for treatment and infection control management. This case study evidentially proves that administrators, quality professionals, and care providers in the Al-Ahsa region have ensured that the healthcare system was able to withstand the pandemic by their response by quickly implementing changes and improvements alongside the efficient use of the $\mathrm{MOH}$ facilities.

\section{Materials and Methods}

The study was conducted in Al-Ahsa, the largest province in the eastern region of the Kingdom of Saudi Arabia. The province's population is estimated to be 1.2 million inhabitants. The $\mathrm{MOH}$ oversees and runs the public not-for-profit governmental healthcare services provided through 10 hospitals and 66 primary health care services [5]. The first case of COVID-19 reported in the Kingdom of Saudi Arabia was on the March 25, 2020, and the Government of Saudi Arabia and the Saudi MOH announced an immediate major lockdown and curfew in all regions of the Kingdom excluding healthcare workers and providers [6].

The major challenge facing the implementation of any major project in healthcare is the lack of human capital trained and experienced in project management. With the rise of the COVID-19 pandemic, the best suggested and implemented practice was to develop distance training and learning sessions in project management for leaders in hospitals to cope with the current situation [7].

As a result, a developed training program was approved and delivered online to build knowledge and capacity for quality and patient safety (QPS) managers. The main theme of this program was based on the concepts of PMI and DMAIC methodology, project charter, requirements, work form, and the project life cycle, 
Fig. 1. Quick-wins project life cycle PMI-

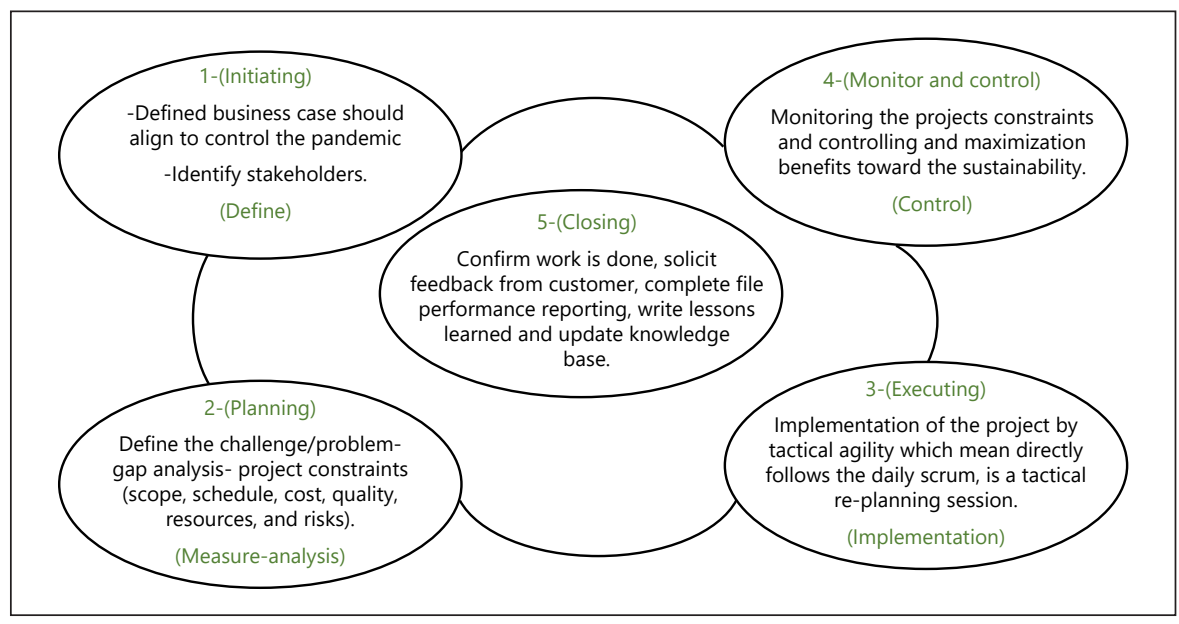
DMAIC approach.

and milestones of these outlines were covered by developing educational video materials.

\section{Design}

This study is designed to be descriptive and retrospective, reflecting on the experience of using the project management approach in the implementation of initiatives to compact COVID-19. The researcher's intention was to use the collaboration approach integrated with PMI and Lean Six Sigma's DMAIC methodologies to implement the initiatives in harmony with the execution team responsible for implementation within the target hospitals, who had limited knowledge and experience of the PMI approach. As the healthcare sector is an agile industry, this indicates the need of a nimble and rapid approach to process and solutions. In addition, health services and processes are extremely complex and sophisticated needing innovative strategies and approaches to process improvement, especially in disaster and crisis circumstances [8].

For Project Management Professional certification, PMI continuously develops and enhances methodology. The PMBOK Sixth Edition developed for Project Management Professional consists of ten knowledge areas and 49 processes categorized in 5 process groups - initiating, planning, execution, monitoring and controlling, and closing.

The Six Sigma approach uses statistical analysis of data to measure and improve the organizations' operational performance. This Six Sigma methodology is known as DMAIC - shown in Figure 1. Some of the most common tool sets of Six Sigma are drawn from both statistics and project management processes. The Six Sigma approach uses statistical analysis of data to measure and improve the organizations' operational performance. The purpose of this integrated approach was to achieve consistency within the project's management and deliverables [9].

The blend between the two concepts and methodologies could be outlined and summarized as following:

- Step 1: define and initiate using Six Sigma: this step includes defining the case and stakeholders.

- Step 2 includes the measurement and analysis steps from the Six Sigma methodology and the planning from PMI. This stage includes defining the problem and associated challenges with it, filling in the gaps, and analyzing the resources, risks, and cost that may be required.
- Step 3 is a combination of the improvement implementation step in the Six Sigma methodology and the execution step from PMI. This step includes the activities of actual implementation and improvement to the processes that were defined, measured, and analyzed.

- Step 4 is about the result of combining the control approach from Six Sigma with monitoring from the PMI methodology. In this step, the improvement team monitors the project and removes all the obstacles and constraints to maximize the benefits in sustaining improvement.

- Step 5 is the final stage of the project, and as with any plan or project, this mainly includes reevaluating the project at every level and ensuring that the improvement was delivered alongside a 360-degree feedback action from all stakeholders to learn lessons for initiating and implementing future improvement projects.

In addition, a facilitation and communications plan was developed between both the Ministry's directorate QPS office and the QPS departments at the 12 participating facilities. At the beginning of the pandemic, training courses in project management and healthcare improvement methods were provided remotely and virtually. The Administration of QPS in Al-Ahsa health affairs contacted providers of different training programs to enhance the skills of QPS's leaders at the hospital level. The first main objectives of this task force were the alignment of recommendations to control COVID-19 cases with the Command-and-Control Center (CCC). The CCC has daily reports and minutes of meetings at the directorate level of the $\mathrm{MOH}$ in the Al-Ahsa region. The second was the enterprise engagement of all related departments at all the region's hospitals, and the third was to define the baseline and the associated challenges. The program started by sending emails to the targeted health facilities that would be participating in this project, requesting one to three suggested initiatives and listing the top priorities and challenges facing the hospitals at the start of the pandemic.

\section{Data Collection}

Data were collected through a standardized report form designed to document a quantitative descriptive of the outcomes and the lessons learned of each initiative. The initiatives that met criteria were implemented in different departments such as the emer- 
Table 1. Challenges domains and selected projects performance

\begin{tabular}{llll}
\hline Domains of alignment & Ranking of selected projects' scope & Projects, \% $(n)$ & Improvement of baselines, \% \\
\hline Work environment & Compliance with updated policy and the procedure & $32.5(13)$ & 81 \\
& Improving practice of support workers & $15(6)$ & 78 \\
& Safe patient internal transfer & $2.5(1)$ & 20 \\
& Monitoring availability of PPEs & $2.5(1)$ & 100 \\
\hline Empowerment of care & Accessibility to the updated protocols & $2.5(1)$ & 100 \\
providers & Update the digital medical document & $2.5(1)$ & 100 \\
& Clinical training and privileges upgrade & $10(4)$ & 100 \\
\hline Improvement of patients' & Auman support to front lines & $7.5(3)$ & 87 \\
experience & Psychosocial support & $20(8)$ & 70.5 \\
\hline Projects closing status & Projects continuing with the pandemic (72.5\%) & Closed projects (25\%) & Rescheduling projects (2.5\%) \\
\hline
\end{tabular}

gency department, inpatient pharmacy, and physiotherapy which were required to meet the challenges between April 26 and May 21,2020 . Each initiative was assigned an implementation team, which worked at every facility facing the previously described challenges, ensuring that they adopted and were fully compliant with the MOH's control protocol for the COVID-19 pandemic while also able to ensure continuity of healthcare provision.

The QPS task force responsible for projectification and the quick-wins program designed a format to secure integration and alignment on all facility levels. Every facility was required to adopt the format guidelines through assignment of a portfolio manager (head of infection control or hospital director), program manager (head of QPS), project manager (head of each department that undertook an initiative), and nominated team members (process owners and providers responsible for provision of care). Initiatives were categorized by the predetermined zones' work environment, health practitioners' empowerment, and improvement of patients' experience.

\section{Results}

In reference to participating 2 specialized care centers and 10 hospitals with a bed capacity ranging from 30 to 500 beds, the researcher concluded that there were several quick wins achieved, and these are summarized as follows:

1. Three of the 43 proposed projects were excluded as they did not fulfill or meet the suggested inclusion criteria.

2. There was clear involvement and buy-in from leadership at almost all the hospitals participating in the initiatives, project domains, and provision of support.

3. There was strong engagement from all the stakeholders associated with all projects, especially patients receiving physical therapy.

Projectification and Quick Wins during the COVID-19 Pandemic
4. The improved processes adopted became the default workflow as a new standard and became the norm.

5. All team members and caregivers felt more able to speak up as they spoke up clearly and openly, which represented an improvement in communication.

6. Processes were improved, targeted, and enabled efficient outcomes.

7. There was significant sharing of experiences, best practices, and lessons learned throughout the healthcare system, which were presented at weekly virtual meetings.

8. Risk consideration and analysis by all project participants improved significantly and served as a precursor to future mode effect analysis.

9. The teams adhered to their plans and agreed timeline, thus avoiding any delays.

10.By understanding when and how, $\mathrm{MOH}$ hospitals were able to significantly improve responsiveness.

11.Stakeholders were able to effectively implement patient-centered concepts and involvement in their therapeutic plans.

Findings from the Projectification Approach in the Health Sector to Control the COVID-19 Pandemic

The domain projects faced the early challenges of needing to align with the recommendations of the CCC and their interpretation of $\mathrm{MOH}$ guidelines. The QPS task force categorized projects into three key domains: work environment, improving patient experience, and care provider empowerment, as shown in Table 1.

Project ranking within each domain, combined with the defined challenges to be projectivized, is as follows: 
1. Domain: work environment, which represented $52.5 \%$ of the program's domains.

Projects: compliance with updated policies and procedures was evident in $32.5 \%$ of the total number of projects. The average improvement above the defined baseline was $81 \%$.

Projects: improved practice by support workers was reported in $15 \%$ of the total number of projects, and the average improvement beyond the defined baseline was $78 \%$.

2. Domain: improving patient's experience during lockdown and curfew - this domain represented $25 \%$ of the study's domains.

Projects: ability to access health service during the period of the pandemic lockdown was demonstrated in $20 \%$ of the total number of domain projects and the reported average improvement above the defined baseline was $87 \%$.

3. Domain: empowerment of care providers - this domain represented $22.5 \%$ of the study's domains.

Projects: the upgrade of clinical training and privileges appeared in $10 \%$ of the total number of projects, with a total average improvement above the defined baseline as $87 \%$.

Projects' completion rate was $72.5 \%$, and $25 \%$ of projects were completed successfully. Two point five percent of the total projects had to be rescheduled.

\section{Discussion/Conclusion}

The study outlined in Table 1 shows that adopting a projectification approach for projects aimed at controlling the COVID-19 pandemic in Al-Ahsa health facilities successfully and converted those projects into quick wins. Adopting lean management processes, standardizing project tools, and eliminating unnecessary communication enhanced both integrated care and team spirit and productivity.

Adopting a projectification approach enhanced control of the pandemic by aligning and integrating the team structure. These findings are supported by Oesterreich et al. [4] in their study. By working together, quality improvement during the COVID-19 pandemic facilitated rapid internal mobilization and a unified response that spanned the entire enterprise. The first domain - work environment and initiatives focused on compliance with updated policies and procedures, safe patient internal transfer, improving support workers' practices, and monitoring the availability of PPEs, was the largest domain equivalent to $52.5 \%$ of the total, and represented an average improvement of $78 \%$ above the defined baseline.

The challenges of our study correspond to a report released by the US Department of Health and Human Services (HHS) based on a survey conducted from March 2020, with one or more administrators from 323 hospitals in 46 states. The hospitals reported that the most significant challenge they faced during the COVID-19 pandemic was "unclear guidance from federal, state, and local authorities, which was confusing to both healthcare staff and the public." Conflicts in guidance on testing, elective procedure deferral, PPE usage, and supply from the national stockpile were noted [10].

The second domain, improvement in patient experience during the lockdown represented $25 \%$ of activity. There was a substantial increase in the utilization of distant clinical visits via telemedicine and medication dispensing - telemedicine increases patient satisfaction [11], decreases costs [12], and enhances the patient experience [13]. Accessibility to health service during the early stages of the pandemic was demonstrated in $20 \%$ of the total number of domain projects, and the average calculated improvement above the defined baseline was reported as $87 \%$.

These results are synonymous with a report released by the US Department of HHS, which concluded that the public had been misinformed. This represented a challenge for hospitals because they had to increase their workload as patients were coming to hospitals unnecessarily and relying on hospitals for education and information [14].

The third domain, empowerment of care providers, represented $22.5 \%$ of domain projects undertaken in the $\mathrm{Al}$-Ahsa region during the pandemic. Projects within this domain focused on upgrading clinical training and privileges and represented approximately $10 \%$ of the total number of domain projects. However, the average improvement above the defined baseline was reported as $87 \%$. These projects were developed to overcome the challenge of maintaining the quality and quantity of both specialized and support personnel. According to Travers et al. [15] in their study of the influence of empowered work environments on the psychological experiences of nursing assistants during COVID-19, nursing assistants who felt empowered tended to perform their duties more effectively and efficiently, which increased their productivity, self-confidence, morale, and job satisfaction and were therefore less likely to leave their jobs. This was evident in all the projects undertaken during the pandemic in Al-Ahsa as workers felt excited and motivated to see
Saudi J Health Syst Res 2022;2:62-67 DOI: $10.1159 / 000521659$
Alsaqer/Abduljawad/Mustafa/ Mohammad 
the results of quality improvement and to participate in improving the healthcare system [15].

We therefore concluded that the projectification approach of enhancing enterprise integration and alignment is one of the most common challenges for any healthcare system on a daily basis and is especially difficult during an epidemic, in response to a disaster and especially during a global pandemic. In summary, the study had some limitations - we relied on initiative managers to self-report progress/updates, and the study excluded primary healthcare as out of scope. Our study concluded that if our healthcare system is faced with similar challenges in the future, similar practices should be established, ensuring that research, experimentation, implementation, and case-control measures are applied.

\section{Acknowledgment}

The author would like to thank Mr. Robert Connor (Head of Al-Ahsa Health Cluster TMO); editing and proof-reading.

The author also would like to thank the leaders and staff of AlAhsa's participating hospitals especially those leading or working in hospital QPS teams.

\section{Statement of Ethics}

The Local Committee for Research Ethics at King Fahd Hospital Al-Hofuf (IRB KFHH No. H-05-HS-065) has revised and approved the study protocol. Informed consent was not required as this study did not involve human subjects or a clinical trial.

\section{Conflict of Interest Statement}

The authors have no conflict of interest to declare.

\section{Funding Sources}

The authors did not receive any funding.

\section{Author Contributions}

Mr. Adnan Ahmed AlSaqer (transformation strategic planner and Accountable Care Organization [ACO] lead for the Al-Ahsa Health Care Cluster): development of the study's concept, design, data acquisition, manuscript preparation, and primary authorship of the study. Prof. Asaad A. Abduljawad (dean of the College of Health Sciences University of Umm Alqura Health Sciences Center, Mekkah, KSA): literature search, manuscript editing, and manuscript review. Dr. Hatim Sid Ahmed Mohammad Mustafa (strategic management manager with the Al-Ahsa Health Cluster, KSA): wrote methodology of the study and drafted the study's introduction. Dr Maha Salam Mohammed (change management lead with the Al-Ahsa Health Cluster, KSA): data analysis and manuscript review.

\section{Data Availability Statement}

Data referenced within this study can be made available by the corresponding author.

\section{References}

1 Guide A, editor. Project management body of knowledge (pmbok ${ }^{\oplus}$ guide). Project Management Institute; 2001.

2 Hodgson D, Fred M, Bailey S, Hall P. The projectification of the public sector. Routledge; 2019.

3 Oesterreich S. [cited 8/6/2021]. Available from: https://hal.archives-ouvertes.fr/hal-01579996/ document.

4 Oesterreich S, Cywinski JB, Elo B, Geube M, Mathur P. Quality improvement during the COVID-19 pandemic. Ccjm. 2020.

$5 \mathrm{MOH}$. 2021. Available from: https://www. moh.gov.sa/Ministry/Statistics/book/Pages/ default.aspx.

6 Kabene SM, Orchard C, Howard JM, Soriano MA, Leduc R. The importance of human resources management in health care: a global context. Hum Resour Health. 2006 Dec;4(1): 20-7.
7 Alrashed S, Min-Allah N, Saxena A, Ali I, Mehmood R. Impact of lockdowns on the spread of COVID-19 in Saudi Arabia. Inform Med Unlocked. 2020;20:100420.

8 Tolf S, Nyström ME, Tishelman C, Brommels M, Hansson J. Agile, a guiding principle for health care improvement? Int J Health Care Qual Assur. 2015 Jun 8;28(5):468-93.

9 Gray J, Anantatmula V. Managing six sigma projects through the integration of six sigma and project management processes. Ijssca. 2009;5(2):127.

10 Grimm CA. Hospital experiences responding to the COVID-19 pandemic: results of a national pulse survey March 23-27, 2020. 2020 [cited 2020 Nov 27]. Available from: https:// oig.hhs.gov/oei/reports/oei-06-20-00300.asp.

11 Sabesan S, Simcox K, Marr I. Medical oncology clinics through videoconferencing: an acceptable telehealth model for rural patients and health workers. Intern Med J. 2012;42(7): $780-5$.
12 Baker LC, Johnson SJ, Macaulay D, Birnbaum $\mathrm{H}$. Integrated telehealth and care management program for medicare beneficiaries with chronic disease linked to savings. Health Aff. 2011;30(9):1689-97.

13 Ramaswamy A, Yu M, Drangsholt S, Ng E, Culligan PJ, Schlegel PN, et al. Patient satisfaction with telemedicine during the $\mathrm{CO}$ VID-19 pandemic: retrospective cohort study. J Med Int Res. 2020;22(9):e20786.

14 Grimm CA. Hospital experiences responding to the COVID-19 pandemic: results of a national pulse survey March 23-27, 2020. Washington, DC: US Department of Health and Human Services OoIG; 2020. p. 1-41.

15 Travers JL, Schroeder K, Norful AA, Aliyu S. The influence of empowered work environments on the psychological experiences of nursing assistants during COVID-19: a qualitative study. BMC Nurs. 2020;19(1):98-12. 